\title{
Investigating light NMSSM pseudoscalar states with boosted ditau tagging
}

\author{
Eric Conte, ${ }^{a}$ Benjamin Fuks, ${ }^{b, c}$ Jun Guo, ${ }^{d, e}$ Jinmian $\mathbf{L i}^{f}$ and Anthony G. Williams ${ }^{f}$ \\ ${ }^{a}$ Groupe de Recherche de Physique des Hautes Énergies (GRPHE), \\ Université de Haute-Alsace, IUT Colmar, \\ 34 rue du Grillenbreit BP 50568, 68008 Colmar Cedex, France \\ ${ }^{b}$ Sorbonne Universités, UPMC Univ. Paris 06, \\ UMR 7589, LPTHE, F-75005, Paris, France \\ ${ }^{c}$ CNRS, UMR 7589, LPTHE, F-75005, Paris, France \\ ${ }^{d}$ State Key Laboratory of Theoretical Physics, Institute of Theoretical Physics, \\ Chinese Academy of Sciences, \\ Beijing 100190, P.R. China \\ ${ }^{e}$ Institut Pluridisciplinaire Hubert Curien/Département Recherches Subatomiques, \\ Université de Strasbourg/CNRS-IN2P3, \\ 23 rue du Loess, F-67037 Strasbourg, France \\ ${ }^{f}$ ARC Centre of Excellence for Particle Physics at the Terascale and CSSM, \\ Department of Physics, University of Adelaide, \\ Adelaide, SA 5005, Australia \\ E-mail: eric.conte@iphc.cnrs.fr, fuks@lpthe.jussieu.fr, \\ hustgj@itp.ac.cn, phyljm@gmail.com, anthony.williams@adelaide.edu.au
}

ABSTRACT: We study a class of realizations of the Next-to-Minimal Supersymmetric Standard Model that is motivated by dark matter and Higgs data, and in which the lightest pseudoscalar Higgs boson mass is smaller than twice the bottom quark mass and greater than twice the tau lepton mass. In such scenarios, the lightest pseudoscalar Higgs boson can be copiously produced at the LHC from the decay of heavier superpartners and will dominantly further decay into a pair of tau leptons that is generally boosted. We make use of a boosted object tagging technique designed to tag such a ditau jet, and estimate the sensitivity of the LHC to the considered supersymmetric scenarios with 20 to $50 \mathrm{fb}^{-1}$ of proton-proton collisions at a center-of-mass energy of $13 \mathrm{TeV}$.

KEYWORDS: Supersymmetry Phenomenology

ARXIV EPRINT: 1604.05394 


\section{Contents}

\section{Introduction 1}

2 Light scalar and pseudoscalar Higgs bosons in the NMSSM 3

2.1 Theoretical framework 3

2.2 Exploration of the NMSSM parameter space 4

2.3 Identification of benchmark scenarios for Run-II LHC studies 9

3 LHC sensitivity to light NMSSM pseudoscalar Higgs bosons decaying to a boosted ditau jet 10

3.1 Event simulation for the NMSSM and boosted ditau tagging 10

3.2 LHC sensitivity to NMSSM light higgsinos decaying to boosted ditau objects 12

4 Conclusion

\section{Introduction}

The Standard Model of particle physics has been proven an extremely successful theory of nature, but it leaves many questions unanswered. It is consequently widely acknowledged as an effective theory obtained from a more fundamental theoretical context still to be observed. Supersymmetric extensions of the Standard Model represent one of the most popular options for new physics and are motivated by the unification of gauge and spacetime symmetries. In addition, they resolve the hierarchy problem inherent to the Standard Model, feature the unification of the gauge couplings at high energy scales and naturally provide an explanation to the presence of dark matter in the universe. By construction, the Higgs sector of a supersymmetric theory is extended with respect to the Standard Model case and contains at least two weak doublets of Higgs superfields (traditionally noted $H_{u}$ and $H_{d}$ ) so that masses for both the up-type and down-type fermions could be generated. Considering the minimal supersymmetric extension of the Standard Model, the so-called Minimal Supersymmetric Standard Model (MSSM) [1, 2], only the $H_{u}$ and $H_{d}$ Higgs supermultiplets are included and the superpotential contains a supersymmetric mass term for these superfields $\mu H_{u} H_{d}$. While the dimensionful parameter $\mu$ should in principle be of the order of the only natural scale of the theory that is either the Planck or the gauge-coupling unification scale, a working spontaneous breaking of the electroweak symmetry demands this parameter to be in the ball park of a few hundreds of GeV. This puzzle is called the ' $\mu$-problem' of the MSSM [3]. On different lines, the discovery of a scalar field with a mass of about $125 \mathrm{GeV}$ and that resembles the Standard Model Higgs boson $[4,5]$ implies either the existence of heavy top squarks or large top squark mixing, which raises questions about the naturalness of the MSSM. 
All these issues can be solved elegantly in the framework of the Next-to-Minimal Supersymmetric Standard Model (NMSSM) [6], where the model includes an additional superfield $S$ that is singlet under the Standard Model gauge group. As a result, the Higgs sector of the model features three neutral scalar states, two neutral pseudoscalar states and one charged state, as well as one singlino (the fermionic component of $S$ ) and two higgsinos (the fermionic components of $H_{u}$ and $H_{d}$ ) fermions that will mix with the gauginos to form five neutralinos. This enriched particle content yields a phenomenology that could be largely different from the MSSM case and that could even accomodate [7] the tantalizing hints of an excess of diphoton events observed in LHC data at a center-of-mass energy of $13 \mathrm{TeV}[8,9]$.

In order to satisfy the stringent constraints on the Standard Model Higgs boson properties derived from LHC measurements [10,11], and in particular those that are put on the Higgs exotic decay modes, phenomenologically viable NMSSM scenarios have to contain a Standard-Model-like Higgs boson with a very small singlet component. Consequently, one given scalar state and one given pseudoscalar state have to be almost purely singlet, so that they couple to the Standard Model only through their small mixing with the $H_{u}$ and $H_{d}$ fields. Furthermore, these singlet fields are weakly constrained by current experimental data and can hence be as light as a few $\mathrm{GeV}$. This setup with two light singlet-like bosons is further motivated by the Peccei-Quinn symmetry limit of the NMSSM where one imposes that the model Lagrangian is invariant under a Peccei-Quinn-like symmetry. This indeed not only allows the NMSSM to solve the strong $C P$-problem [12], but also yields a very light pseudoscalar singlet state $A_{1}$. Such a prediction has spurred an intense phenomenological activity over the last years [13-20], with a particular focus on processes where light $A_{1}$ pairs are produced from the cascade decays of heavier Higgs bosons. Pioneering works have investigated final state systems made of four jets issued from the fragmentation of $b$-quarks $H \rightarrow A_{1} A_{1} \rightarrow b \bar{b} b \bar{b}$ [21], four leptonically or hadronically decaying tau leptons $H \rightarrow A_{1} A_{1} \rightarrow \tau^{+} \tau^{-} \tau^{+} \tau^{-}$[22-24], four muons $H \rightarrow A_{1} A_{1} \rightarrow \mu^{+} \mu^{-} \mu^{+} \mu^{-}$[25] or of one pair of muons and one pair of tau leptons $H \rightarrow A_{1} A_{1} \rightarrow \mu^{+} \mu^{-} \tau^{+} \tau^{-}$[26]. It has been moreover shown that the discovery of such decay channels would consist of a no-lose theorem for a direct evidence of the NMSSM [27, 28]. This has consequently opened the path for dedicated NMSSM searches in LHC collision data at a center-of-mass energy of $8 \mathrm{TeV}$ [29-31]. Upper limits on the production cross sections related to the four taus, two taus and two muons, and four muons decay modes of the heavy Higgs boson of 4.5-10.3 pb, 0.72-2.33 pb and of about $1 \mathrm{fb}$ have been respectively derived.

In this work, we study Higgs data constraints on the NMSSM and show that phenomenologically viable scenarios feature configurations in which the lightest pseudoscalar $A_{1}$ is dominantly produced from the decays of neutralino states [32-34]. The LHC constraints on these scenarios are still both rather weak and very model dependent. In particular, $\tilde{\chi}_{i}^{0} \rightarrow A_{1} \tilde{\chi}_{j}^{0}$ decays often lead to the production of boosted $A_{1}$ particles that are difficult to detect due to the collimation of their decay products into a single object, regardless of the mass splitting between the two neutralinos $\tilde{\chi}_{i}^{0}$ and $\tilde{\chi}_{j}^{0}$. We explore in section 2 the parameter space of the NMSSM and investigate specific scenarios compatible with the above-mentioned Higgs requirements and featuring a light pseudoscalar state $A_{1}$, with a 
focus on cases where its mass is of at least twice as large as the tau lepton mass and smaller than twice the mass of the $b$-quark. In our process for constructing such scenarios, we additonally impose dark matter considerations on the lightest supersymmetric partner. We then investigate, in section 3, the sensitivity of the current LHC run at $13 \mathrm{TeV}$ to such scenarios and show that they could be detected through the analysis of a signature comprised of a single lepton, a ditau-tagged jet and missing transverse energy. To this aim, we make use of a ditau tagging technique that has been developed in the context of the Higgs [35-37] and that we have supplemented to a multivariate analysis dedicated to the tagging of the signal. Our conclusions are summarized in section 4.

\section{Light scalar and pseudoscalar Higgs bosons in the NMSSM}

\subsection{Theoretical framework}

The NMSSM is constructed by augmenting the MSSM superfield content by one superfield $S$ that is a singlet under $\mathrm{SU}(3)_{c} \times \mathrm{SU}(2)_{L} \times \mathrm{U}(1)_{Y}$. After the breaking of supersymmetry (and the consequent breaking of the electroweak symmetry), the scalar component of $S$ mixes with the Higgs degrees of freedom, whilst the fermionic component of $S$, dubbed the singlino, mixes with the two remaining higgsino states and the gauginos. As for any softly broken supersymmetric theory, the NMSSM is specified by its superpotential and its supersymmetry-breaking Lagrangian. The superpotential reads

$$
W_{\mathrm{NMSSM}}=-L H_{d} \mathbf{y}_{\mathbf{e}} E-Q H_{d} \mathbf{y}_{\mathbf{d}} D+Q H_{u} \mathbf{y}_{\mathbf{u}} U+\lambda S H_{u} H_{d}+\frac{1}{3} \kappa S^{3},
$$

where all indices are omitted for brevity, where $Q$ and $L$ denote the weak doublets of quark and lepton superfields and where $U, D$ and $E$ are the up-type quark, down-type quark and lepton weak-singlet superfields, respectively. In addition, we have introduced the $3 \times 3$ Yukawa matrices $\mathbf{y}$ and the $\lambda$ and $\kappa$ parameters that drive the couplings of $S$. In particular, once the scalar component $s$ of the singlet superfield gets a vacuum expectation value $\langle s\rangle=v_{s} / \sqrt{2}$, an effective $\mu$-term is generated,

$$
\mu_{\mathrm{eff}}=\frac{1}{\sqrt{2}} \lambda v_{s}
$$

which solves the MSSM $\mu$-problem. In the expression of eq. (2.1), we have imposed that the superpotential satisfies a $\mathbb{Z}_{3}$ symmetry so that any dimensionful term allowed by the gauge symmetry is forbidden.

The soft supersymmetry breaking Lagrangian contains mass terms for all scalar $\left(\mathbf{m}_{\tilde{\mathbf{Q}}}^{2}\right.$, $\mathbf{m}_{\tilde{\mathbf{U}}}^{2}, \mathbf{m}_{\tilde{\mathbf{D}}}^{2}, \mathbf{m}_{\tilde{\mathbf{L}}}^{2}, \mathbf{m}_{\tilde{\mathbf{E}}}^{2}, m_{H_{u}}^{2}, m_{H_{d}}^{2}$ and $\left.m_{s}^{2}\right)$ and gaugino $\left(M_{1}, M_{2}\right.$ and $\left.M_{3}\right)$ fields, as well as trilinear interaction terms $\left(\mathbf{A}^{\mathbf{u}}, \mathbf{A}^{\mathbf{d}}, \mathbf{A}^{\mathbf{e}}, A_{\lambda}\right.$ and $\left.A_{\kappa}\right)$ sharing the form of the superpotential,

$$
\begin{aligned}
\mathcal{L}_{\text {soft }}= & -\frac{1}{2}\left[M_{1} \tilde{B} \tilde{B}+M_{2} \tilde{W} \tilde{W}+M_{3} \tilde{g} \tilde{g}+\text { h.c. }\right] \\
& -\mathbf{m}_{\tilde{\mathbf{Q}}}^{\mathbf{2}} \tilde{q}^{\dagger} \tilde{q}-\mathbf{m}_{\tilde{\mathbf{U}}}^{\mathbf{2}} \tilde{u}^{\dagger} \tilde{u}-\mathbf{m}_{\tilde{\mathbf{D}}}^{\mathbf{2}} \tilde{d}^{\dagger} \tilde{d}-\mathbf{m}_{\tilde{\mathbf{L}}}^{\mathbf{2}} \tilde{\ell}^{\dagger} \tilde{\ell}-\mathbf{m}_{\tilde{\mathbf{E}}}^{\mathbf{2}} \tilde{e}^{\dagger} \tilde{e}-m_{H_{u}}^{2} h_{u}^{\dagger} h_{u}-m_{H_{d}}^{2} h_{d}^{\dagger} h_{d}-m_{s}^{2} s^{\dagger} s \\
& +\left[-\mathbf{y}^{\mathbf{u}} \mathbf{A}^{\mathbf{u}} \tilde{u}^{\dagger} \tilde{q} h_{u}+\mathbf{y}^{\mathbf{d}} \mathbf{A}^{\mathbf{d}} \tilde{d}^{\dagger} \tilde{q} h_{d}+\mathbf{y}^{\mathbf{e}} \mathbf{A}^{\mathbf{e}} \tilde{e} \tilde{e}^{\dagger} \tilde{\ell} h_{d}-\lambda A_{\lambda} h_{u} h_{d} s-\frac{1}{3} \kappa A_{\kappa} s^{3}+\text { h.c. }\right],
\end{aligned}
$$


where $q, \ell, u, d, e, h_{u}, h_{d}$ and $s$ denote the scalar components of the $Q, L, U, D, E, H_{u}, H_{d}$ and $S$ superfields, respectively, and $\tilde{B}, \tilde{W}$ and $\tilde{g}$ the gauginos associated with the $\mathrm{U}(1)_{Y}$, $\mathrm{SU}(2)_{L}$ and $\mathrm{SU}(3)_{C}$ gauge groups. All indices are again understood for clarity.

In order to reduce the number of free parameters, we assume that all parameters related to the (s)fermion sector are flavor-conserving and universal at the grand unification scale. Introducing the common scalar mass $m_{0}$, the common gaugino mass $m_{1 / 2}$ and the common trilinear coupling $A_{0}$, we have

$$
\begin{aligned}
\mathbf{m}_{\tilde{\mathbf{Q}}}^{\mathbf{2}} & =\mathbf{m}_{\tilde{\mathbf{U}}}^{\mathbf{2}}=\mathbf{m}_{\tilde{\mathbf{D}}}^{\mathbf{2}}=\mathbf{m}_{\tilde{\mathbf{L}}}^{\mathbf{2}}=\mathbf{m}_{\tilde{\mathbf{E}}}^{\mathbf{2}}=m_{0}^{2} \mathbf{1}_{3 \times 3}, \quad M_{1}=M_{2}=M_{3}=m_{1 / 2}, \\
\mathbf{A}^{\mathbf{u}} & =\mathbf{A}^{\mathbf{d}}=\mathbf{A}^{\mathbf{e}}=A_{0} \mathbf{1}_{3 \times 3},
\end{aligned}
$$

where $\mathbf{1}_{3 \times 3}$ stands for the identity matrix in flavor space.

In this framework, the Higgs sector is defined by the soft parameters $A_{\lambda}$ and $A_{\kappa}$ that we fix at the grand unification scale, and by the $\lambda, \kappa, \tan \beta$ and $\mu_{\text {eff }}$ parameters that are provided at the electroweak scale, $\tan \beta$ being the ratio of the vacuum expectation values of the neutral components of the two Higgs doublets $h_{u}$ and $h_{d}$.

\subsection{Exploration of the NMSSM parameter space}

In the previous section, we have defined a parameterization of the NMSSM in terms of nine free parameters,

$$
m_{0}, \quad m_{1 / 2}, \quad A_{0}, \quad A_{\lambda}, \quad A_{\kappa}, \quad \lambda, \quad \kappa, \quad \tan \beta \quad \text { and } \mu_{\text {eff }},
$$

the first five parameters being defined at the grand unification scale and the last four parameters being defined at the electroweak scale. We supplement to these the parameters related to the Standard Model sector,

$$
\begin{aligned}
\alpha_{s}\left(m_{Z}\right) & =0.1172, & G_{F} & =1.1663910^{-5} \mathrm{GeV}^{-2}, \\
m_{Z} & =91.187 \mathrm{GeV}, \quad m_{t}^{\text {pole }}=173.1 \mathrm{GeV}, & \alpha\left(m_{Z}\right) & =1 / 127.92, \\
m_{\tau} & =1.777 \mathrm{GeV} . & & m_{b}\left(m_{b}\right)=4.214 \mathrm{GeV},
\end{aligned}
$$

The QCD interaction strength is computed from the value of the strong coupling constant at the $Z$-pole $\alpha_{s}\left(m_{Z}\right)$ and the three independent electroweak inputs, whose values are taken from the Particle Data Group review [38], are chosen to be the Fermi constant $G_{F}$, the $Z$-boson mass $m_{Z}$ and the electromagnetic coupling evaluated at the $Z$-pole $\alpha\left(m_{Z}\right)$. Finally, the third generation fermion sector is defined by the pole mass of the top quark $m_{t}^{\text {pole }}$, the running $\overline{\mathrm{MS}}$ mass of the bottom quark $m_{b}\left(m_{b}\right)$ evaluated at the $m_{b}$ scale and the tau mass $m_{\tau}$, all other fermion masses being neglected. All the couplings and masses appearing in the NMSSM Lagrangian can be subsequently numerically calculated, using in particular the relations determined by the minimization of the scalar potential.

For our exploration of the NMSSM parameter space, we use the NMSSMTools package [39-41] and perform a scan over the parameters given in eq. (2.5). The ranges in which the parameters are allowed to vary are given in table 1 , and for scanned each point, we 


\begin{tabular}{|c|c|c|c|c|}
\hline$m_{0}$ & $m_{1 / 2}$ & $A_{0}$ & $A_{\lambda}$ & $A_{\kappa}$ \\
\hline$[400,2000] \mathrm{GeV}$ & {$[1000,2000] \mathrm{GeV}$} & {$[-5000,-1000] \mathrm{GeV}$} & {$[-500,500] \mathrm{GeV}$} & {$[0,300] \mathrm{GeV}$} \\
\hline
\end{tabular}

\begin{tabular}{|c|c|c|c|}
\hline$\lambda$ & $\kappa$ & $\tan \beta$ & $\mu$ \\
\hline$[0.2,0.5]$ & {$[0.01,0.2]$} & {$[1.5,15]$} & {$[100,350] \mathrm{GeV}$} \\
\hline
\end{tabular}

Table 1. Parameterization of the NMSSM parameter space explored in this work. We indicate the ranges in which the different parameters have been allowed to vary. In the first table, the parameters are provided at the grand unification scale while in the second table, they are given at the electroweak scale.

impose a set of constraints that allows us to accept it or reject it. Additionally to theoretical considerations such as obtaining a physical spectrum that does not exhibit any tachyonic state or preventing the appearance of a Landau pole below the grand unification scale, we impose limits on the Higgs sector and on the supersymmetric particles derived from collider searches at LEP, at the Tevatron and at the LHC. We moreover verify the consistency of the selected points with dark matter data.

In order to accomodate a Higgs boson with properties close to those expected in the case of the Standard Model, the mixing between the singlet state $s$ and the two Higgs doublets $h_{u}$ and $h_{d}$ has to be small. In this case, the tree-level masses of the $C P$-even and $C P$-odd singlet-like Higgs bosons are mostly given by the corresponding entries in the $3 \times 3$ scalar and pseudoscalar squared mass matrices $\mathcal{M}_{S}^{2}$ and $\mathcal{M}_{P}^{2}$,

$$
\begin{aligned}
& \left(\mathcal{M}_{S}^{2}\right)_{33}=\lambda A_{\lambda} \frac{v^{2} \sin 2 \beta}{2 v_{s}}+\frac{\kappa v_{s}}{\sqrt{2}}\left(A_{\kappa}+2 \sqrt{2} \kappa v_{s}\right), \\
& \left(\mathcal{M}_{P}^{2}\right)_{33}=\lambda A_{\lambda} \frac{v^{2} \sin 2 \beta}{2 v_{s}}+\frac{\kappa v_{s}}{\sqrt{2}}\left(\frac{\sqrt{2} \lambda v^{2} \sin 2 \beta}{v_{s}}-3 A_{\kappa}\right),
\end{aligned}
$$

where the vacuum expectation value $v$ is defined, at the tree level, by

$$
v^{2}=\frac{4 m_{Z}^{2}}{g_{1}^{2}+g_{2}^{2}},
$$

$g_{1}$ and $g_{2}$ denoting the hypercharge and weak coupling constants. In this work, we focus on the phenomenology of scenarios in which the lightest pseudoscalar state $A_{1}$ is mostly singlet-like, so that $m_{A_{1}}^{2} \approx\left(\mathcal{M}_{P}^{2}\right)_{33}$, and where its mass is heavier than twice the tau lepton mass and lighter than twice the bottom quark mass, $m_{A_{1}} \in\left[2 m_{\tau}, 2 m_{b}\right]$. As a consequence, a cancellation between the different terms of eq. (2.8) should be in place. Since the natural size of each term is of about the electroweak scale squared $\mathcal{O}\left(10^{4}\right) \mathrm{GeV}^{2}$, the mass of the lightest singlet-like scalar state, that is approximately given by eq. (2.7) in the absence of a too large singlet-doublet mixing, will be smaller than $125 \mathrm{GeV}$. It turns out that the second scalar Higgs particle $\mathrm{H}_{2}$ has to be identified with the boson discovered during the first run of the LHC. Its mass $m_{H_{2}}$ can moreover be generally compatible with the observed value of $125 \mathrm{GeV}$, the presence of the lighter singlet-like $H_{1}$ state helping to increase it through mixing effects [42]. We thus impose in our scan that $m_{H_{2}}$ lies in the $[122.1,128.1] \mathrm{GeV}$ mass window, such a large range allowing us to account for the various sources of uncertainties both on the experimental result and on the theoretical prediction. 
Beside its mass, the first run of the LHC has allowed one to measure a lot of properties of the Higgs boson with high precision. One specific ensemble of such measurements consists of the so-called Higgs signal strengths $\mu_{X, Y}$ that are defined as the ratios of predicted rates in a particular production channel $X$ and decay mode $Y$ of the Higgs boson in a given new physics theory (being here the NMSSM) to the Standard Model expectation,

$$
\mu_{X, Y}=\epsilon_{X, Y} \frac{\sigma_{X}}{\sigma_{X}^{\mathrm{SM}}} \frac{\mathrm{BR}(h \rightarrow Y)}{\operatorname{BR}^{\mathrm{SM}}(h \rightarrow Y)} .
$$

The $\epsilon_{X, Y}$ factor in the above expression is related to the acceptance and efficiency of the analyses under consideration, and could be different in a new physics context and in the Standard Model. Such differences are however usually assumed to be mild and to largely cancel in the ratio. The signal strengths moreover depend on the Higgs production cross sections in the $X$ channel $\left(\sigma_{X}^{\mathrm{SM}}\right.$ and $\left.\sigma_{X}\right)$ and branching ratios associated with the $h \rightarrow Y$ decay mode $\left(\mathrm{BR}(h \rightarrow Y)\right.$ and $\left.\mathrm{BR}^{\mathrm{SM}}(h \rightarrow Y)\right)$ both in the Standard Model and in the NMSSM. Making use of the NMSSM signal strengths predicted by NMSSMTools and the experimental measurements at the LHC [10, 11], we construct a $\chi^{2}$ quantity [43]

$$
\begin{aligned}
\chi_{Y}^{2}= & a_{Y}\left(\mu_{\mathrm{ggF}, Y}-\hat{\mu}_{\mathrm{ggF}, Y}\right)^{2}+2 b_{Y}\left(\mu_{\mathrm{ggF}, Y}-\hat{\mu}_{\mathrm{ggF}, Y}\right)\left(\mu_{\mathrm{VBF}+\mathrm{VH}, Y}-\hat{\mu}_{\mathrm{VBF}+\mathrm{VH}, Y}\right) \\
& +c_{Y}\left(\mu_{\mathrm{VBF}+\mathrm{VH}, Y}-\hat{\mu}_{\mathrm{VBF}+\mathrm{VH}, Y}\right)^{2},
\end{aligned}
$$

where the 'ggF', 'VBF' and 'VH' indices respectively refer to the gluon-fusion, vectorboson fusion and Higgs-Strahlung Higgs production mechanisms. The $\hat{\mu}_{X, Y}, a_{Y}, b_{Y}$ and $c_{Y}$ parameters resulting from the fit of the LHC Higgs measurements are taken from the LiLith-1.1.3 program using the 15.09 database [44], and we require $\chi_{Y}^{2}<6.18$ so that our NMSSM predictions are compatible at the $2 \sigma$ level with data.

All the points satisfying the requirements introduced so far are represented in figure 1 as a function of the parameters defining the NMSSM Higgs sector. More precisely, we show results in the $(\lambda, \kappa),\left(\tan \beta, \mu_{\mathrm{eff}}\right)$ and $\left(A_{\lambda}, A_{\kappa}\right)$ planes in which correlations can be observed. Each represented point refers to a particle spectrum free from any tachyonic state and in which the second lightest Higgs boson $H_{2}$ has a mass comprised in the $[122.1,128.1] \mathrm{GeV}$ range and yields a good $\chi^{2}$ fit of the measured signal strengths. We indicate the scenarios for which the pseudoscalar mass is smaller than $30 \mathrm{GeV}$ by gray points, while the green points correspond to cases in which $2 m_{\tau}<m_{A_{1}}<2 m_{b}$. It turns out that small $\lambda$ and $\kappa$ values are generally favored, which results in a light $A_{1}$ state as given by eq. (2.8). On different lines, the $\tan \beta$ and $\mu_{\mathrm{eff}}$ parameters are strongly correlated, and a large (small) value of $\tan \beta$ leads to a small (large) $\mu_{\text {eff }}$ value. This feature originates from imposing that the $H_{2}$ scalar boson is Standard-Model-like. Finally, an approximately linear correlation between the $A_{\lambda}$ and $A_{\kappa}$ parameters is shown in the lower left panel of the figure. This arises from the singlet-doublet mixing that is more conveniently assessed when the scalar components of the Higgs fields are rotated to the so-called Higgs basis $\left(H_{\mathrm{SM}}, H^{\prime}, S^{\prime}\right)$ by means of an appropriate $\mathrm{U}(2)$ transformation [45-47]. This basis choice has the advantage that only one of the non-singlet fields, $H_{\mathrm{SM}}$, acquires a vacuum expectation value $v$ and features Standard-Model-like interactions with the Standard Model fermions and gauge bosons. 
The second non-singlet field $H^{\prime}$ has thus a vanishing vacuum expectation value and the third basis element $S^{\prime}$ remains a pure singlet state. In the Higgs basis, the size of the singletdoublet mixing is given by the $H_{\mathrm{SM}}-S^{\prime}$ element of the squared mass matrix $\mathcal{M}_{S}^{\prime 2}[12,48]$,

$$
\left(\mathcal{M}_{S}^{\prime 2}\right)_{13}=\frac{\lambda v}{\sqrt{2}}\left(2 \mu_{\mathrm{eff}}-\left(A_{\lambda}+\sqrt{2} \kappa v_{s}\right) \sin 2 \beta\right)
$$

In order for $H_{2}$ to be Standard-Model-like and thus mostly equivalent to $H_{\mathrm{SM}}$ in the Higgs basis, $\left(\mathcal{M}_{S}^{\prime 2}\right)_{13}$ must be small, which leads to

$$
A_{\lambda} \sim \frac{2 \mu_{\mathrm{eff}}}{\sin 2 \beta}-\frac{2 \kappa \mu_{\mathrm{eff}}}{\lambda}
$$

However, in the NMSSM parameter space region of interest, we have $\sin 2 \beta \ll \lambda / \kappa$. Consequently, only the first term of the right-hand side of eq. (2.13) matters. Moreover, $\mu_{\mathrm{eff}}$ and $\tan \beta$ are correlated, as shown in the upper right panel of figure 1, and both $A_{\lambda}$ and $A_{\kappa}$ are connected to the Higgs spectrum, as shown, e.g., by eq. (2.7) and eq. (2.8). Although deriving the exact relation between the $A_{\lambda}$ and $A_{\kappa}$ parameters that are defined in our setup at the grand unification scale is a complex task due to renormalization group running, an approximatively linear relation can be derived and is indeed observed in the figure.

As a result of the above constraints, the typical spectrum exhibited by the scenarios selected during our scan of the NMSSM parameter space features a lightest neutralino state $\tilde{\chi}_{1}^{0}$ that is mainly singlino-like, whilst the next two neutralinos $\tilde{\chi}_{2}^{0}$ and $\tilde{\chi}_{3}^{0}$ are close in mass and higgsino-like. Once produced, these two heavier neutralinos can decay, sometimes with a large branching ratio, into a final state system comprised of a singlino $\tilde{\chi}_{1}^{0}$ and a pseudoscalar Higgs boson $A_{1}$. In the lower right panel of figure 1, we present the dependence of these branching ratios on the mass difference between the higgsino states (with masses being approximatively taken as $\mu_{\text {eff }}$ ) and the singlino. This shows that an important $A_{1}$ production rate can be achieved in the region where the spectrum is compressed, i.e., when the mass difference between the higgsinos and the singlino is small. As soon as $\mu_{\text {eff }}-m_{\tilde{\chi}_{1}^{0}} \gtrsim 90 \mathrm{GeV}$, the $\tilde{\chi}_{i}^{0} \rightarrow Z \tilde{\chi}_{1}^{0}$ channel opens and quickly dominates. We have included in our results the $\tilde{\chi}_{3}^{0} \rightarrow A_{1} \tilde{\chi}_{2}^{0}$ decay contributions. Although the phase space for such a decay process is very limited as both higgsino states are close in mass (so that the $A_{1}$ decay products are soft and very hard to detect), the final-state $\tilde{\chi}_{2}^{0}$ higgsino will further decay into an $A_{1}$ particle that will be energetic enough to leave observable tracks in a detector.

Additionally to the constraints that we have imposed so far, we moreover restrict the properties of the lightest neutralino $\tilde{\chi}_{1}^{0}$ so that it could be a good dark matter candidate. To this aim, we first impose that the associated relic density abundance $\Omega h^{2}$ is compatible with the latest Planck results [49],

$$
0.107<\Omega h^{2}<0.131
$$

where the allowed range for $\Omega h^{2}$ includes theoretical uncertainties on the predictions. Since the $\tilde{\chi}_{1}^{0}$ particle is of a singlino-dominated nature, a small $\mu-m_{\tilde{\chi}_{1}^{0}}$ mass splitting, or 

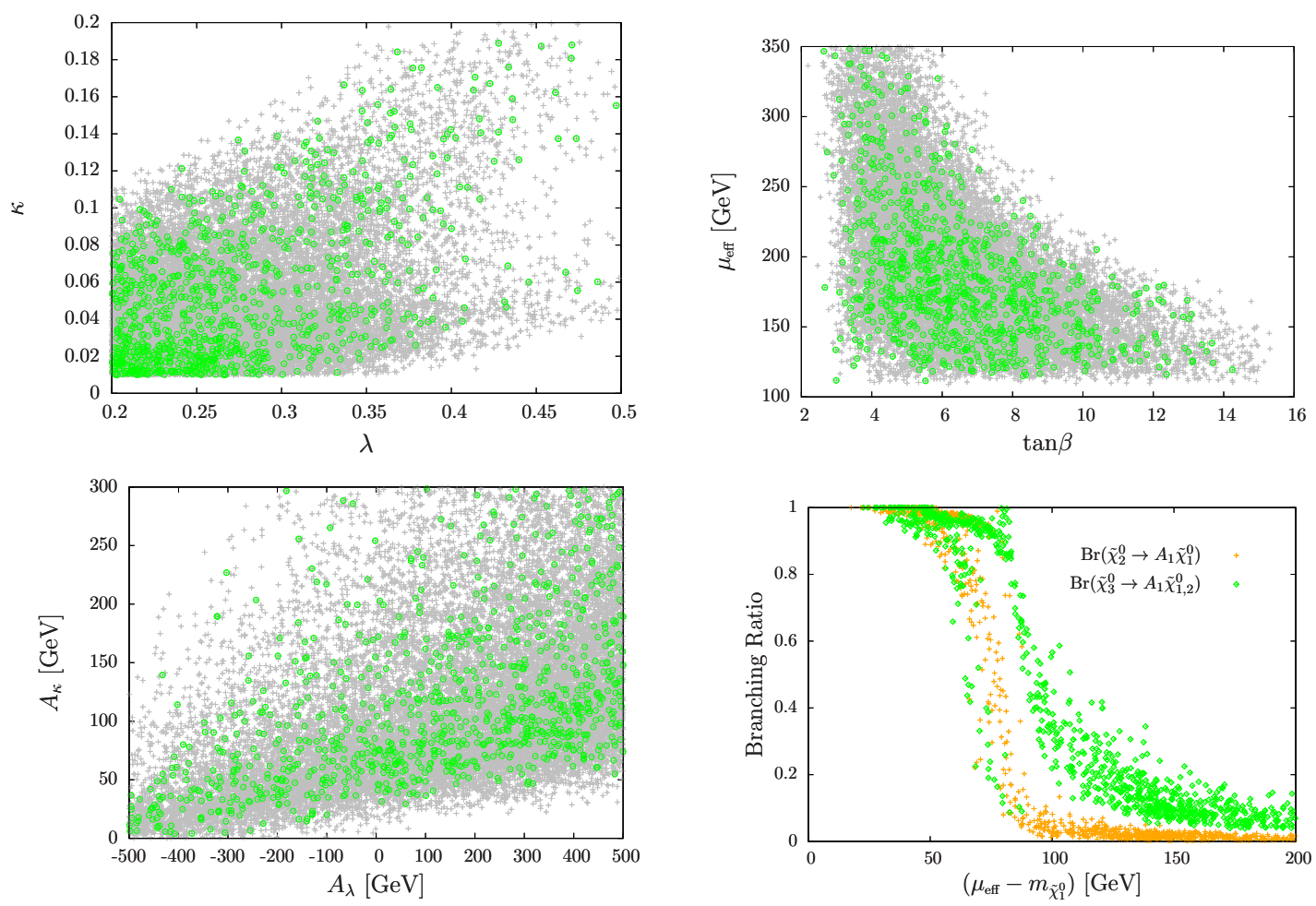

Figure 1. Benchmark NMSSM scenarios selected in our scanning procedure of the NMSSM parameter space, presented in the $(\lambda, \kappa)$ (upper left panel), $\left(\tan \beta, \mu_{\text {eff }}\right)$ (upper right panel) and $\left(A_{\lambda}, A_{\kappa}\right)$ (lower left panel) planes. All models for which a physical NMSSM spectrum at the electroweak scale is found are represented, under the condition that the second lightest scalar state $\mathrm{H}_{2}$ is StandardModel-like. We distinguish the cases where $m_{A_{1}}<30 \mathrm{GeV}$ (gray) and where $2 m_{\tau}<m_{A_{1}}<2 m_{b}$ (green). In the lower right panel, we depict the dependence of the second and third neutralino branching fractions into a pseudoscalar $A_{1}$ state on the compressivity of the spectrum defined as $\mu_{\mathrm{eff}}-m_{\tilde{\chi}_{1}^{0}}$, for all points satisfying $2 m_{\tau}<m_{A_{1}}<2 m_{b}$

equivalently a large higgsino-singlino mixing, is required for efficient enough dark matter (co)annihilation processes. This is illustrated on the left panel of figure 2 in which we study the relations between the lightest neutralino relic density as computed with the MicrOMEgAs package $[50,51]$ and the $\mu-m_{\tilde{\chi}_{1}^{0}}$ splitting. We observe that the scenarios selected in our scan are spread among three regions of the parameter space. In the heavy dark matter region for which $m_{\tilde{\chi}_{1}^{0}} \gtrsim m_{H_{2}} / 2$, dark matter annihilation proceeds via an $s$-channel $Z$-boson exchange diagram that highly depends on the higgsino fraction of the lightest neutralino $\tilde{\chi}_{1}^{0}$. As a consequence, a relic density in agreement with Planck data implies that the mass splitting $\mu-m_{\tilde{\chi}_{1}^{0}}$ is at most of about $70 \mathrm{GeV}$. In contrast, in the two other regions, the so-called Higgs funnel region for which $m_{\tilde{\chi}_{1}^{0}} \sim m_{H_{2}} / 2$ and $Z$-boson funnel region for which $m_{\tilde{\chi}_{1}^{0}} \sim m_{Z} / 2$, larger splittings are allowed as the dark matter annihilation cross section is enhanced by the presence of resonant diagrams that allow to recover a relic density in agreement with eq. (2.14). 

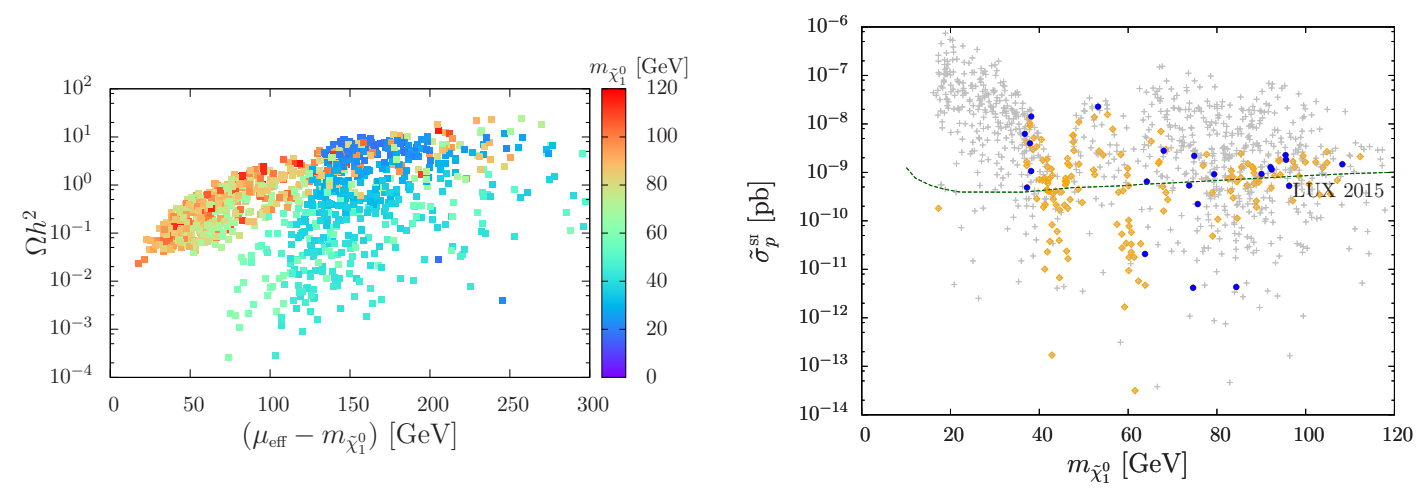

Figure 2. Dark matter constraints that have been imposed during our NMSSM parameter space scan. We show, in the left panel, the dark matter relic density $\Omega h^{2}$ as a function of the mass splitting between the lightest (singlino-dominated) neutralino $\tilde{\chi}_{1}^{0}$ and the higgsinos, whose mass is of about $\mu_{\text {eff }}$. We additionally provide information on the lightest neutralino mass (color code). On the right panel, we present the spin-independent dark-matter-proton scattering cross section scaled by $\Omega h^{2} / 0.119$ as a function of the lightest neutralino mass. We focus on scenarios for which $2 m_{\tau}<m_{A_{1}}<2 m_{b}$ (gray) that additionally feature $\Omega h^{2}<0.131$ (orange) or $0.107<\Omega h^{2}<0.131$ (blue). We indicate the LUX bounds from 2015 by a dark green line.

We next focus on the spin-independent dark-matter-proton scattering cross section,

$$
\tilde{\sigma}_{p}^{\mathrm{SI}}=\sigma_{p}^{\mathrm{SI}} \frac{\Omega h^{2}}{0.119},
$$

and compare the NMSSM predictions obtained with MicrOmegas to LUX data [52]. The results are presented in the right panel of figure 2 , in which we demonstrate that many models in all three regions can survive all considered dark matter constraints. The heavy dark matter region is additionally expected to be easier to probe within future dark matter direct detection experiments that will further constrain the scattering cross section $\tilde{\sigma}_{p}^{\mathrm{SI}}$.

\subsection{Identification of benchmark scenarios for Run-II LHC studies}

All the NMSSM scenarios compliant with the constraints imposed so far exhibit common features. The sfermions are typically lying in the multi- $\mathrm{TeV}$ region are are thus out of the reach of the LHC from the direct search standpoint, at least with an assumed luminosity of $50-100 \mathrm{fb}^{-1}$. In contrast, the singlino and higgsino states are in general light (with masses below $400-500 \mathrm{GeV}$ ) and the gauginos heavy (with masses greater than $1 \mathrm{TeV}$ ). As a results, the only superpartners that could be produced with a sufficiently large rate, and thus observed, consist of the second and third neutralinos (or equivalently, the higgsino-like neutralinos) and the lightest chargino. As already discussed in the previous subsection, the singlet-like Higgs boson $H_{1}$ has a mass smaller than about $100 \mathrm{GeV}$, while the second scalar state $H_{2}$ is identified with the Standard-Model Higgs boson. The lightest pseudoscalar state $A_{1}$ is singlet-like with a mass satisfying $2 m_{\tau}<m_{A_{1}}<2 m_{b}$ and all other Higgs particles are beyond $1 \mathrm{TeV}$.

In order to study the discovery prospects for these scenarios at the LHC, we focus on a specific benchmark point that is defined in terms of the nine parameters given in the upper 


\begin{tabular}{|c|c|c|c|c||c|c|c|c|}
\hline$m_{0}$ & $m_{1 / 2}$ & $A_{0}$ & $A_{\lambda}$ & $A_{\kappa}$ & $\lambda$ & $\kappa$ & $\tan \beta$ & $\mu$ \\
\hline $1215.3 \mathrm{GeV}$ & $1872.8 \mathrm{GeV}$ & $-4112.1 \mathrm{GeV}$ & $301.1 \mathrm{GeV}$ & $204.8 \mathrm{GeV}$ & 0.317 & 0.122 & 12.2 & $121.3 \mathrm{GeV}$ \\
\hline
\end{tabular}

\begin{tabular}{|c|c|c||c||c|c||c|c|c|}
\hline$m_{\tilde{\chi}_{1}^{0}}$ & $m_{\tilde{\chi}_{2}^{0}}$ & $m_{\tilde{\chi}_{3}^{0}}$ & $m_{\tilde{\chi}_{1}^{ \pm}}$ & $m_{A_{1}}$ & $m_{A_{2}}$ & $m_{H_{1}}$ & $m_{H_{2}}$ & $m_{H_{3}}$ \\
\hline $75.7 \mathrm{GeV}$ & $-135.3 \mathrm{GeV}$ & $149.2 \mathrm{GeV}$ & $124.2 \mathrm{GeV}$ & $5.5 \mathrm{GeV}$ & $1538 \mathrm{GeV}$ & $93.8 \mathrm{GeV}$ & $125.9 \mathrm{GeV}$ & $1538 \mathrm{GeV}$ \\
\hline
\end{tabular}

\begin{tabular}{|c||c|c||c||c|}
\hline $\operatorname{Br}\left(\tilde{\chi}_{2}^{0} \rightarrow A_{1} \tilde{\chi}_{1}^{0}\right)$ & $\operatorname{Br}\left(\tilde{\chi}_{3}^{0} \rightarrow A_{1} \tilde{\chi}_{1}^{0}\right)$ & $\operatorname{Br}\left(\tilde{\chi}_{3}^{0} \rightarrow A_{1} \tilde{\chi}_{2}^{0}\right)$ & $\operatorname{Br}\left(A_{1} \rightarrow \tau \tau\right)$ & $\operatorname{Br}\left(H_{2} \rightarrow A_{1} A_{1}\right)$ \\
$98.9 \%$ & $12.9 \%$ & $87.1 \%$ & $93.6 \%$ & $4.2 \%$ \\
\hline
\end{tabular}

\begin{tabular}{|c|c||c|c|}
\hline$\mu_{g g \rightarrow H, \gamma \gamma}$ & $\mu_{\mathrm{VBF}, V V^{*}}$ & $\Omega h^{2}$ & $\sigma_{p}^{\mathrm{SI}}$ \\
\hline 1.06 & 1.02 & 0.107 & $2.46 \times 10^{-10} \mathrm{pb}$ \\
\hline
\end{tabular}

Table 2. Representative NMSSM benchmark scenario satisfying all Higgs and dark matter constraints imposed in our scan. The scenario is defined in the upper part of the table, in which we recall that the parameters of the left part of the table are given at the grand unification scale while those of its right part are defined at the electroweak scale. The light state masses are given in the middle panel of the table, while relevant branching ratios and obsevable results are given in its lower parts.

panel of table 2. The light part of the resulting mass spectrum is presented in the second panel of the table, while relevant branching ratios and Higgs and dark matter observables are shown in its two lower panels. In the context of such a benchmark scenario, the new physics processes yielding the largest production cross section consist of the production of a pair of (neutral or charged) higgsinos at the LHC. By a virtue of their dominant decay modes, current LHC searches are not sensitive to their associated signature. Neutralino production leads to the further production of pseudoscalar Higgs bosons that next decay into pairs of boosted taus, while the lightest chargino decays into an off-shell $W$-boson and the singlino so that no LHC bound is expected from the charged higgsino side [53]. Following the procedure developed in ref. [54, 55], we recast all relevant Run-I LHC analyses. We find the CMS search for same-sign dilepton [56] is the most sensitive to NMSSM benchmark points such as the one depicted above. However, the relevant production rates are almost two orders of magnitude lower than the signal cross section that is excluded at the $95 \%$ confidence level.

In addition, the $H_{2} \rightarrow A_{1} A_{1}$ branching ratio reaches about $4 \%$, a result compatible with Higgs current data. Although this decay mode has been actively searched for [29-31] as it consists of an irrefutable proof of the realization of an NMSSM scenario [27, 28], we focus instead, in the next section, on a novel discovery mode for a light NMSSM pseudoscalar based on boosted ditau tagging.

\section{LHC sensitivity to light NMSSM pseudoscalar Higgs bosons decaying to a boosted ditau jet}

\subsection{Event simulation for the NMSSM and boosted ditau tagging}

To determine the LHC sensitivity to the class of NMSSM models introduced in the previous section, we analyze Monte Carlo simulations of proton-proton collisions at a center-ofmass energy of $13 \mathrm{TeV}$ as they could occur at the LHC collider at CERN. Hard-scattering 
signal and background simulations rely on the MADGRAPH5_AMC@NLO program [57] that contains an NMSSM implementation. The latter, that has not been described in any earlier publication, is generated by the FEYNRULES package [58] and its superspace module [59] that automatically produce a UFO library [60] that can be employed by MADGRAPH5_AMC@NLO for event generation, following the strategy of ref. [61]. The FEYNRuLES model includes a more general version of the superpotential of eq. (2.1) and the soft supersymmetry-breaking Lagrangian of eq. (2.3) where the $\mathbb{Z}_{3}$ symmetry that we have imposed in this work is not included and where intergenerational sfermion mixings are allowed. Although such mixings are not handled by NmssmTools, they are compatible with the Supersymmetry Les Houches Accord conventions [62, 63] in which their implementation consists of optional requirements. The translation of the output spectrum files produced by NMSsmTools to files compliant with the NMSSM UFO is nevertheless immediate as this only necessitates to increase the size of $2 \times 2$ mixing matrices to $6 \times 6$ matrices with zero entries whenever two different sfermion generations are concerned. The validation of the NMSSM implementation of FEYNRULES has been extensively performed during the 2009 Les Houches workshop on TeV collider physics [64], and thousands of supersymmetric processes have been considered to this aim.

The decays of the produced hard particles and the matching of the parton-level hard events to a parton shower and hadronization infrastructure have been performed in the context of the Pythia 6 package [65], that is interfaced to Tauola [66, 67] for handling tau lepton decays. In this framework, any tau lepton polarization effect is neglected. Finally, we have simulated the response of the ATLAS detector by means of the DeLPHES 3 program [68], that internally reconstructs objects on the basis of the anti- $k_{T}$ jet algorithm [69] as implemented in the FASTJET software [70].

Since tau leptons dominantly decay hadronically with a corresponding branching ratio of about $65 \%$, the development of efficient related tagging techniques is a very important task in particular with respect to Higgs precision measurements [71] and new physics searches [72]. Jets originating from pure QCD subprocesses and from the hadronic decay of a tau lepton are mainly distinguished from each other by the number of charged tracks inside the jet and the jet energy density profile. The properties specific to a tau jet however turn out to be preserved in the case of a boosted object comprised of two hadronically decaying tau leptons that could arise from the decay of a heavier state. Motivated by such considerations, we have designed an analysis strategy allowing us to detect the signature of boosted pseudoscalar NMSSM Higgs bosons produced from the decay of heavier higgsino states at the LHC. This relies on the tagging of boosted ditau objects via a multivariate method [73] that makes use of the number of tracks inside the ditau jet, the ratio $f_{\text {cent }}=E^{(0.1)} / E^{(0.2)}$ where $E^{(0.1)}\left(E^{(0.2)}\right)$ is the total calorimetric energy deposit in a cone of radius $R=0.1(0.2)$ centered on the jet direction, the transverse momentum of the hardest track inside a cone of radius $R=0.2$ centered on the jet direction computed relatively to the jet $p_{T}$, the $p_{T}$-weighted sum of the angular distances of all tracks inside the jet, the maximum angular distance in the transverse plane between any track lying inside a cone of radius $R=0.2$ centered on the jet direction and the jet direction, the track-based jet invariant mass and the ratio of the jet transverse momentum to the jet invariant mass. 
This strategy can furthermore be improved to gain sensitivity to leptonically decaying taus (within a boosted ditau object) as well. The produced leptons are indeed unlikely to be isolated, so that they could be captured by a selection involving the ratio of the electromagnetic to hadronic calorimetric deposits. Additionally, we also consider in our multivariate tagging technique the $N$-subjettiness variable $\tau_{21}=\tau_{2} / \tau_{1}$ that allows one order to resolve the substructure of the ditau jet $[74,75]$, with $\tau_{N}$ being defined by

$$
\tau_{N}=\frac{\sum_{k} \min \left\{\Delta R_{1, k}, \Delta R_{2, k}, \ldots, \Delta R_{N, k}\right\}}{\sum_{k} p_{T, k} R_{0}} .
$$

In this expression, the summations have to be considered upon all jet constituents, $R_{0}=0.4$ is the jet cone size parameter in the original jet clustering algorithm and $\Delta R_{I, k}$ denotes the distance in the transverse plane between the subjet candidate $I$ and the jet constituent $k$.

All the variables that we have introduced to tag a ditau boosted object strongly depend on the object energy, as it is illustrated on figure 3 for two representative jet energies of $50 \mathrm{GeV}$ and $200 \mathrm{GeV}$ and the $f_{\text {cent }}$ and $\tau_{21}$ variables. We compare the spectra that would be obtained when jets solely originate from up quarks (red dashed curves), charmed quarks (blue dash-dotted curves), bottom quarks (green dotted curves), a single tau lepton (brown dash-dotted curves) and a pseudoscalar NMSSM Higgs boson (black plain curves). The properties of ditau boosted objects are different from the single tau jet and the purely QCD jet cases, so that there exist handles for discriminating them. To this aim, we use a boosted decision tree (BDT) technique that uses all the variables presented in this section. The BDT is trained in the context of jets with specific energies of $50 \mathrm{GeV}$ and $200 \mathrm{GeV}$ and the correlations between the obtained boosted ditau object tagging efficiency and the QCD jet or single tau jet mistagging rates are shown in figure 4. Jets issued from the fragmentation of light quarks are always harder to distinguish from ditau boosted objects, as their properties are similar to the ditau case (see figure 3 ). The corresponding rejection power is particularly small when the jet energy is smaller. Taking as a benchmark a tagging efficiency $\epsilon_{S}=50 \%$, a rejection power $\epsilon_{B}^{-1}$ of only 50 is found for $50 \mathrm{GeV}$ jets, this number increasing to 500 for $200 \mathrm{GeV}$ jets.

\subsection{LHC sensitivity to NMSSM light higgsinos decaying to boosted ditau objects}

In order to estimate the sensitivity of the LHC to the class of NMSSM scenarios under consideration, we study the associated production of a neutralino and a chargino, followed by a neutralino decay into a pseudoscalar Higgs boson and a chargino decay into a far off-shell $W$-boson,

$$
p p \rightarrow \tilde{\chi}_{1}^{ \pm} \tilde{\chi}_{j}^{0} \rightarrow\left(W^{*} \tilde{\chi}_{1}^{0}\right)\left(A_{1} \tilde{\chi}_{1}^{0}\right)
$$

This process features a large cross section, as this was already the case in the MSSM [76-81], and we further impose the off-shell $W$-boson to decay leptonically so that fully hadronic backgrounds can be suppressed. In our simulation, we use next-to-leading signal cross sections that are derived with ProsPino [82] and that mostly agree with the most precise results involving soft-gluon resummation [79-81]. The dominant associated sources 

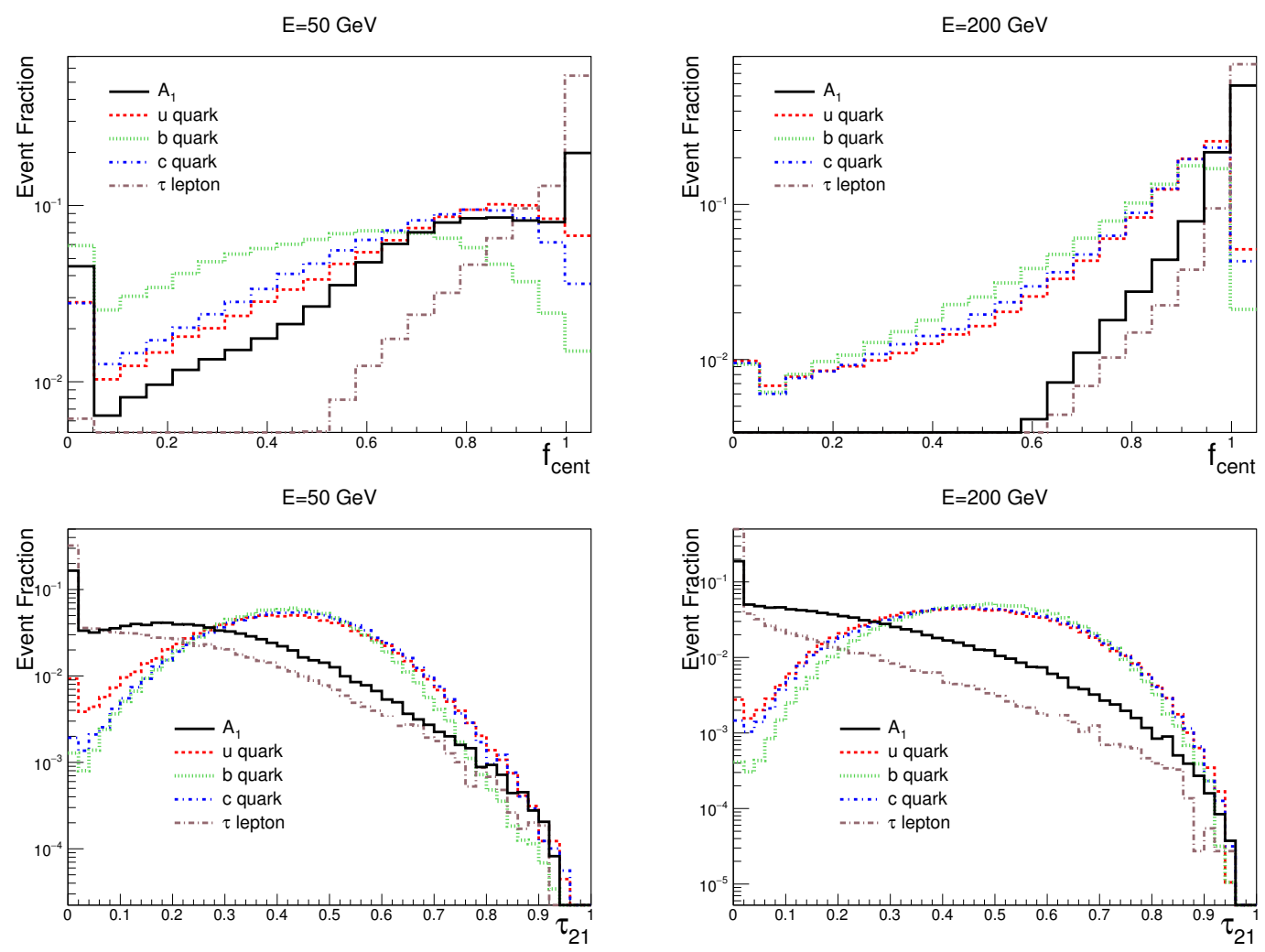

Figure 3. $f_{\text {cent }}$ (upper figures) and $\tau_{21}$ (lower figures) distributions for jets originating from the fragmentation of up quarks (red dashed curves), charmed quarks (blue dash-dotted curves), bottom quarks (green dotted curves), a single tau lepton (brown dash-dotted curves) and a pseudoscalar NMSSM Higgs boson (black plain curves). Two jet energies of $50 \mathrm{GeV}$ (left) and $200 \mathrm{GeV}$ (right) are considered.
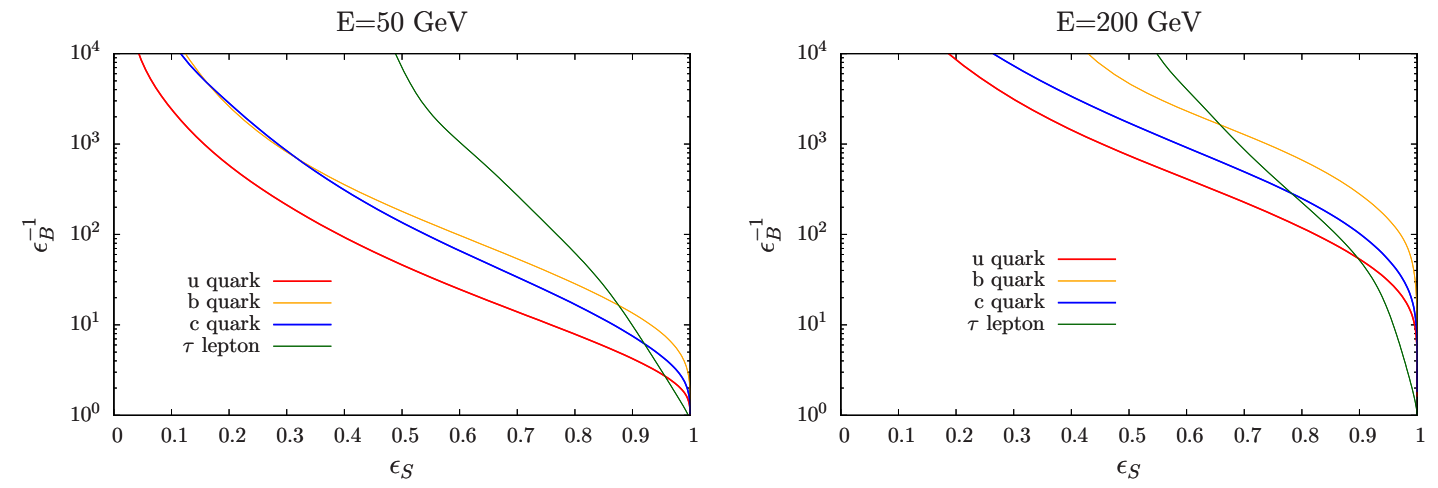

Figure 4. Dependence of the quark and tau lepton rejection powers on the boosted ditau object tagging efficiency for jets of $50 \mathrm{GeV}$ (left) and $200 \mathrm{GeV}$ (right). 
of background consist of events issued from the production of a (leptonically decaying) $W$-boson in association with jets, of top-antitop systems and of diboson systems that all give rise to final states comprised of a single lepton, missing energy and hard jets. We generate events exhibiting at least one jet at the matrix element level and in which the hard-scattering lepton and jets have a transverse momentum $p_{T}>10 \mathrm{GeV}$ and $20 \mathrm{GeV}$ respectively, together with a pseudorapidity satisfying $|\eta|<2.5$. We normalize the topantitop and diboson event samples to the measured [83] and next-to-leading order [84] cross section values respectively, and we make use of the leading-order $W$-boson plus jet fiducial cross section as returned by MADGRAPH5_AMC@NLO to which a next-to-leading order $K$-factor of 1.4 is included [57].

Since signal events feature a final state comprised of a single lepton, a boosted ditau object issued from the decay of a pseudoscalar $A_{1}$ particle and missing energy, we preselect events by requiring that the final state contains exactly one isolated lepton, at least one jet and we veto the presence of $b$-tagged jets. The resulting signal and background cross sections before $\left(\sigma^{13}\right)$ and after $\left(\sigma^{\text {pre }}\right)$ the preselection at $13 \mathrm{TeV}$ LHC are given in table 3 . At this stage of our analysis, background rates are of about three orders of magnitude larger than typical signal cross sections. In the NMSSM scenarios under consideration, the spectrum generally features a mass splitting $\Delta M=\mu_{\text {eff }}-m_{\tilde{\chi}_{1}^{0}}$ between the lightest singlino-like neutralino and the heavier neutral and charged higgsino states of at most $100 \mathrm{GeV}$ (see the lower right panel of figure 1), so that signal events generally exhibit final state systems for which the lepton and jet transverse momenta and the missing transverse energy are of about $50 \mathrm{GeV}$. It is consequently not straightforward to design appropriate selections to enhance the signal over background ratio by only means of the kinematical properties of the signal. The mass splitting $\Delta M$ also determines the energy $E\left(A_{1}\right)$ that is typically carried by the pseudoscalar Higgs bosons $A_{1}$ originating from the decays of the higgsino-like neutralinos. Since $E\left(A_{1}\right)$ is in general much larger than the $A_{1}$ mass, the pseudoscalar decay products (two tau leptons) turn out to be highly collimated. This is illustrated, for the benchmark scenario introduced in section 2.3, on figure 5 where we present the pseudoscalar Higgs boson $A_{1}$ energy spectrum (left panel) and the distribution of the angular distance, in the transverse plane, between the two tau leptons issued from the $A_{1}$ decays (right panel). In figure 6, we study the details of the hadronic activity in the signal events. In the left panel of the figure, we present the distribution of the number of jets $N_{j}$ characterizing the signal events. Although only one jet (the boosted ditau object) is expected from the partonic process, initial state radiation allows the $N_{j}$ spectrum to extent to larger values. The bulk of the events however features at most two jets, while the leading jet is in general the boosted ditau object (in $70 \%$ of the cases for the benchmark scenario of section 2.3). This last property is depicted on the right panel of figure 6 where we present, on the basis of the Monte Carlo truth, the ranking of the $A_{1}$ jet once the $p_{T}$ ordering of the jets is imposed.

As a consequence of these considerations, we further select signal events on the basis of a multivariate analysis that uses as inputs the number of jets $N_{j}$, the transverse momenta of the two leading jets $p_{T}^{j_{1}}$ and $p_{T}^{j_{2}}$, the transverse momentum of the lepton $p_{T}^{\ell}$, the invariant mass of the leading jet, the amount of missing energy $\mathbb{E}_{T}$, the angular distance 

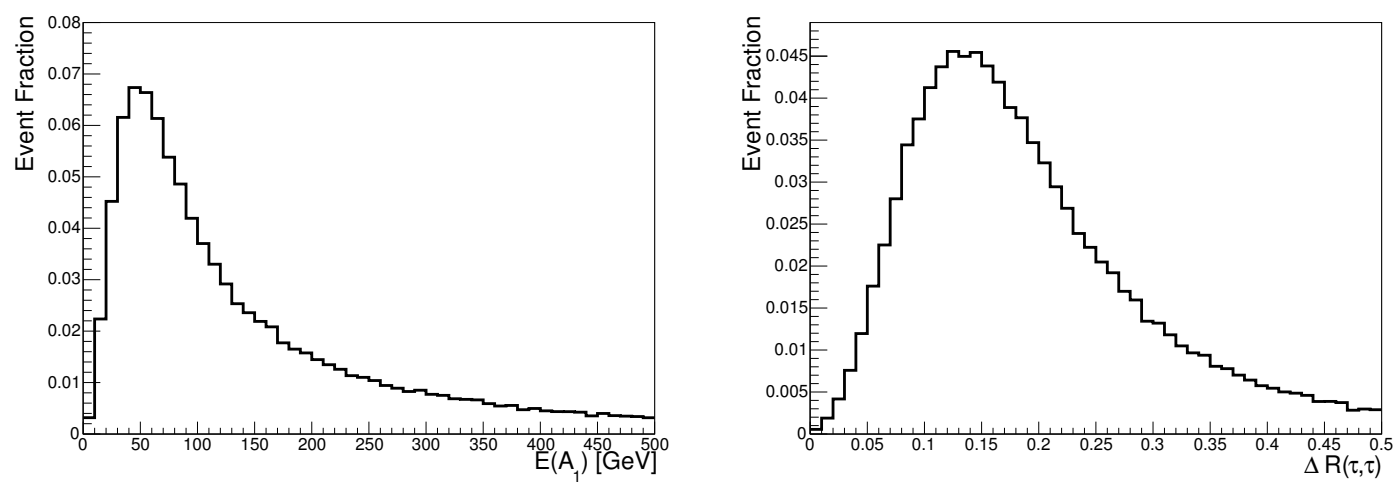

Figure 5. Energy spectrum of the pseudoscalar Higgs bosons $A_{1}$ issued from the decay of neutralino states (left) and the angular distance in the transverse plane between the two taus originating from the $A_{1}$ decays (right) in the context of signal events. Both distributions have been calculated for the NMSSM benchmark scenario introduced in section 2.3 and are normalized to 1.

\begin{tabular}{|c|c|ccc|}
\hline & $\tilde{\chi}^{ \pm} \tilde{\chi}^{0}$ signal & $W$ plus jets & Top pair production & Diboson production \\
\hline$\sigma^{13}$ & $3.38 \mathrm{pb}$ & $8452 \mathrm{pb}$ & $825 \mathrm{pb}$ & $159.3 \mathrm{pb}$ \\
\hline$\sigma^{\text {pre }}$ & $0.42 \mathrm{pb}$ & $4313 \mathrm{pb}$ & $62.9 \mathrm{pb}$ & $29.2 \mathrm{pb}$ \\
\hline
\end{tabular}

Table 3. Signal and background cross section before $\left(\sigma^{13}\right)$ and after $\left(\sigma^{\text {pre }}\right)$ event preselection at $13 \mathrm{TeV}$ LHC.
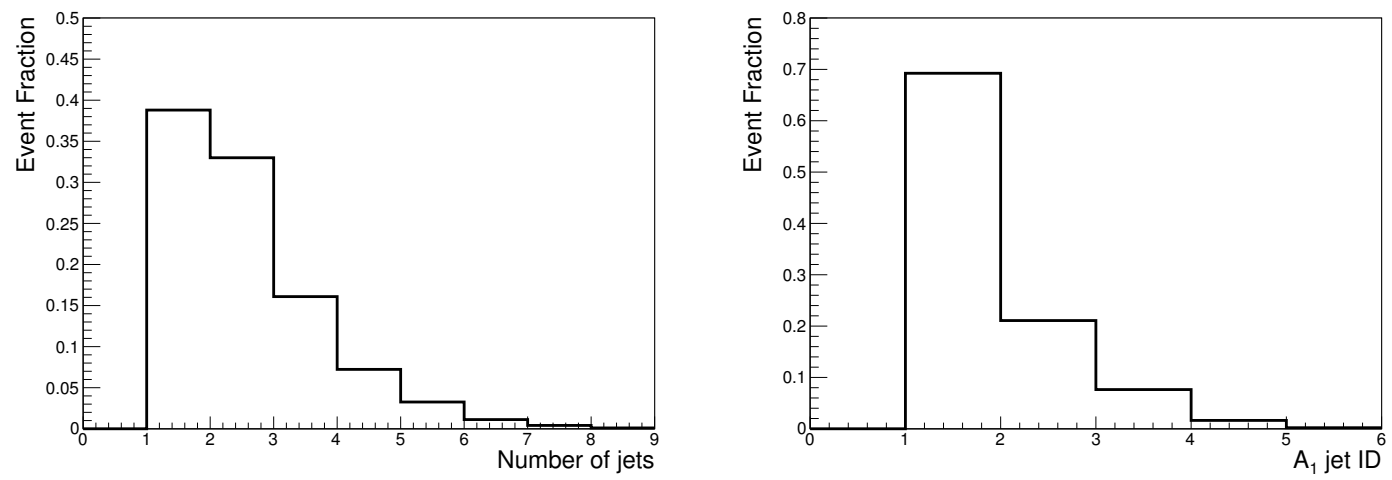

Figure 6. The distribution in the number of jets (left) and the ranking of the boosted ditau jet associated with the $A_{1}$ particle (right) in the case of signal events and in the context of the benchmark scenario introduced in section 2.3. Both distributions are normalized to 1. 

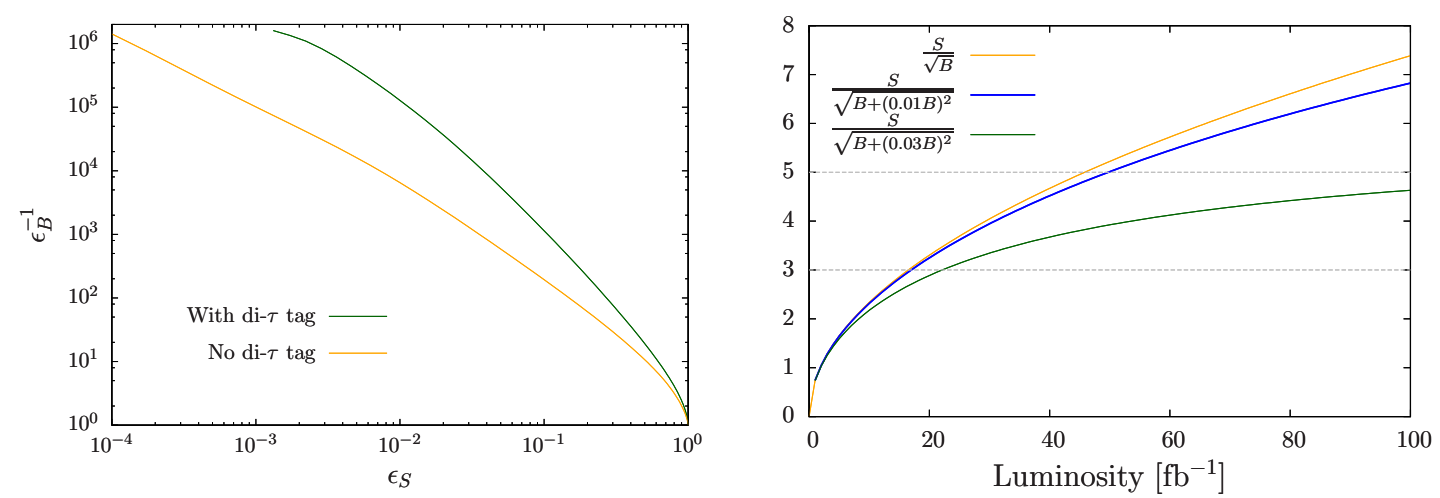

Figure 7. Left: relations between the signal selection efficiency and background rejection rate obtained by means of our multivariate analysis technique. The effect of the tagging of the leading jet as a boosted ditau object is either included (dark green) or not (orange). Right: signal significance obtained with our analysis as a function of the luminosity and for different treatments of the systematic uncertainties on the background.

in the azimuthal direction with respect to the beam between the lepton and the missing momentum $\Delta \phi_{\ell, \mathbf{E}_{T}}$, and the angular distance in the transverse plane between the lepton and the leading jet $\Delta R\left(\ell, j_{1}\right)$. In addition, we also include in our analysis the reconstructed $W$-boson transverse mass $m_{T}^{W}$ that would be obtained when considering that all the missing transverse energy is connected to a $W$-boson decay,

$$
M_{T}^{W}=\sqrt{2 p_{T}^{\ell} \mathbb{E}_{T}\left[1-\cos \Delta \phi_{\ell, \mathbb{E}_{T}}\right]}
$$

and the final state stranverse mass $m_{T 2}[85,86]$. Both these latter variables are expected to provide a handle to efficiently suppress the dominant $W$ background. Moreover, assuming that the boosted ditau object is identified with the leading jet, the next-to-leading jet is expected to be softer in the signal case than in the background case, the lepton and missing energy tend to be not correlated in the signal case as there are several sources of missing energy, and both the $M_{T}^{W}$ and $M_{T 2}$ variables are distributed towards smaller values for the signal as the considered signature is free from any on-shell $W$ boson. The analysis can finally be improved after imposing that the leading jet is a boosted ditau object.

Applying a selection on the BDT response, we can derive the dependence of the background rejection rate $\epsilon_{B}^{-1}$ on the signal selection efficiency $\epsilon_{S}$, as shown in the left panel of figure 7 . We consider two different setups in which the boosted ditau object feature of the signal is accounted for (dark green) or not (orange), and we observe that an efficiency of about one percent can be obtained together with a background rejection rate of $10^{4}\left(\gtrsim 10^{5}\right)$ when the ditau tagging is ignored (included). Optimizing the selection on the BDT output, the resulting signal fiducial cross section is of $3.1 \mathrm{fb}$, while the dominant $W$-boson plus jet and diboson background component ones are of $16.4 \mathrm{fb}$ and $0.9 \mathrm{fb}$ respectively. Moreover, top-antitop events turn to contribute negligibly, with a fiducial cross section smaller than $0.5 \mathrm{fb}$. We calculate the associated signal significance $\sigma_{\text {signal }}$ as a function of the luminosity 
for different treatment of the systematic uncertainties on the background $\Delta B$,

$$
\sigma_{\text {signal }}=\frac{S}{\sqrt{B+(\Delta B)^{2}}},
$$

and show the results on the right panel of the figure. In this expression, $S$ and $B$ denote the number of selected signal and background events respectively. Assuming a systematic uncertainty on the background at the percent level, a $3 \sigma$ hint for the class of NMSSM scenarios considered in this work could be observed at the early stage of the LHC Run-II, while a $5 \sigma$ discovery could be expected with an integrated luminosity of at least $50 \mathrm{fb}^{-1}$.

\section{Conclusion}

It has been recently shown that the discovery of a light pseudoscalar state could be a direct evidence for the next-to-minimal realization of supersymmetry in nature. As a result, many studies have been dedicated to the investigation of the discovery potential associated with such particles. Most existing works focus on heavier Higgs decay probes, although light pseudoscalar states could also be copiously produced from neutralino decays.

In this work, we have explored the NMSSM parameter space and impose Higgs and dark matter constraints on the construction of viable scenarios. We have found that many of such scenarios include pseudoscalar Higgs bosons with a mass comprised in the $\left[2 m_{\tau}, 2 m_{b}\right]$ window. In this case, the golden discovery mode consists of the production of an associated chargino-neutralino pair that further decays into a boosted ditau jet (connected to a pseudoscalar decay), a single lepton and missing energy. We study a typical reference scenario an investigate the sensitivity of the LHC Run-II to the corresponding signal. By means of a multivariate analysis and a boosted ditau object tagging method, we have found that the background could be rejected at a very large level so that a $3 \sigma$ hint for the signal is expected within the first $13 \mathrm{TeV}$ data, and that a $5 \sigma$ discovery could be envisaged for a luminosity of more than about $50 \mathrm{fb}^{-1}$.

\section{Acknowledgments}

This work has ben supported in parts by the Théorie LHC France initiative of the CNRS and a joint CNRS-CAS doctoral grant. The work of JL and AGW is supported by the Australian Research Council through the Centre of Excellence for Particle Physics at the Terascale CE110001004.

Open Access. This article is distributed under the terms of the Creative Commons Attribution License (CC-BY 4.0), which permits any use, distribution and reproduction in any medium, provided the original author(s) and source are credited.

\section{References}

[1] H.P. Nilles, Supersymmetry, Supergravity and Particle Physics, Phys. Rept. 110 (1984) 1 [INSPIRE]. 
[2] H.E. Haber and G.L. Kane, The Search for Supersymmetry: Probing Physics Beyond the Standard Model, Phys. Rept. 117 (1985) 75 [INSPIRE].

[3] J.E. Kim and H.P. Nilles, The $\mu$-problem and the strong CP-problem, Phys. Lett. B 138 (1984) 150 [INSPIRE].

[4] ATLAS collaboration, Observation of a new particle in the search for the Standard Model Higgs boson with the ATLAS detector at the LHC, Phys. Lett. B 716 (2012) 1 [arXiv: 1207.7214] [INSPIRE].

[5] CMS collaboration, Observation of a new boson at a mass of $125 \mathrm{GeV}$ with the CMS experiment at the LHC, Phys. Lett. B 716 (2012) 30 [arXiv:1207.7235] [INSPIRE].

[6] U. Ellwanger, C. Hugonie and A.M. Teixeira, The Next-to-Minimal Supersymmetric Standard Model, Phys. Rept. 496 (2010) 1 [arXiv:0910.1785] [INSPIRE].

[7] U. Ellwanger and C. Hugonie, A $750 \mathrm{GeV}$ Diphoton Signal from a Very Light Pseudoscalar in the NMSSM, arXiv:1602.03344 [INSPIRE].

[8] ATLAS collaboration, Search for resonances decaying to photon pairs in 3.2 $\mathrm{fb}^{-1}$ of $\mathrm{pp}$ collisions at $\sqrt{s}=13 \mathrm{TeV}$ with the ATLAS detector, ATLAS-CONF-2015-081 (2015).

[9] CMS collaboration, Search for new physics in high mass diphoton events in proton-proton collisions at $\sqrt{s}=13 \mathrm{TeV}$, CMS-PAS-EXO-15-004 (2015).

[10] CMS collaboration, Precise determination of the mass of the Higgs boson and tests of compatibility of its couplings with the standard model predictions using proton collisions at 7 and $8 \mathrm{TeV}$, Eur. Phys. J. C 75 (2015) 212 [arXiv:1412.8662] [INSPIRE].

[11] ATLAS collaboration, Measurements of the Higgs boson production and decay rates and coupling strengths using pp collision data at $\sqrt{s}=7$ and $8 \mathrm{TeV}$ in the ATLAS experiment, Eur. Phys. J. C 76 (2016) 6 [arXiv:1507.04548] [InSPIRE].

[12] D.J. Miller, R. Nevzorov and P.M. Zerwas, The Higgs sector of the next-to-minimal supersymmetric standard model, Nucl. Phys. B 681 (2004) 3 [hep-ph/0304049] [INSPIRE].

[13] R. Dermisek and J.F. Gunion, Escaping the large fine tuning and little hierarchy problems in the next to minimal supersymmetric model and $h \rightarrow$ aa decays, Phys. Rev. Lett. 95 (2005) 041801 [hep-ph/0502105] [INSPIRE].

[14] R. Dermisek and J.F. Gunion, The NMSSM Close to the R-symmetry Limit and Naturalness in $h \rightarrow$ aa Decays for $m_{a}<2 m_{b}$, Phys. Rev. D 75 (2007) 075019 [hep-ph/0611142] [INSPIRE].

[15] U. Ellwanger, J.F. Gunion and C. Hugonie, Difficult scenarios for NMSSM Higgs discovery at the LHC, JHEP 07 (2005) 041 [hep-ph/0503203] [INSPIRE].

[16] A. Djouadi et al., Benchmark scenarios for the NMSSM, JHEP 07 (2008) 002 [arXiv: 0801.4321] [INSPIRE].

[17] J. Cao, F. Ding, C. Han, J.M. Yang and J. Zhu, A light Higgs scalar in the NMSSM confronted with the latest LHC Higgs data, JHEP 11 (2013) 018 [arXiv:1309.4939] [INSPIRE].

[18] N.-E. Bomark, S. Moretti, S. Munir and L. Roszkowski, A light NMSSM pseudoscalar Higgs boson at the LHC redux, JHEP 02 (2015) 044 [arXiv:1409.8393] [INSPIRE].

[19] N.-E. Bomark, S. Moretti and L. Roszkowski, Detection prospects of light NMSSM Higgs pseudoscalar via cascades of heavier scalars from vector boson fusion and Higgs-strahlung, arXiv: 1503.04228 [INSPIRE]. 
[20] C.T. Potter, Natural NMSSM with a Light Singlet Higgs and Singlino LSP, Eur. Phys. J. C 76 (2016) 44 [arXiv: 1505.05554] [INSPIRE].

[21] M. Almarashi and S. Moretti, Reinforcing the no-lose theorem for NMSSM Higgs discovery at the LHC, Phys. Rev. D 84 (2011) 035009 [arXiv:1106.1599] [InSPIRE].

[22] J.R. Forshaw, J.F. Gunion, L. Hodgkinson, A. Papaefstathiou and A.D. Pilkington, Reinstating the 'no-lose' theorem for NMSSM Higgs discovery at the LHC, JHEP 04 (2008) 090 [arXiv: 0712.3510] [INSPIRE].

[23] A. Belyaev, S. Hesselbach, S. Lehti, S. Moretti, A. Nikitenko and C.H. Shepherd-Themistocleous, The Scope of the 4 tau Channel in Higgs-strahlung and Vector Boson Fusion for the NMSSM No-Lose Theorem at the LHC, arXiv:0805.3505 [INSPIRE].

[24] D.G. Cerdeno, P. Ghosh and C.B. Park, Probing the two light Higgs scenario in the NMSSM with a low-mass pseudoscalar, JHEP 06 (2013) 031 [arXiv:1301.1325] [INSPIRE].

[25] A. Belyaev, J. Pivarski, A. Safonov, S. Senkin and A. Tatarinov, LHC discovery potential of the lightest NMSSM Higgs in the $H_{1} \rightarrow a_{1} a_{1} \rightarrow 4 \mu$ channel, Phys. Rev. D 81 (2010) 075021 [arXiv: 1002.1956] [INSPIRE].

[26] M. Lisanti and J.G. Wacker, Discovering the Higgs with Low Mass Muon Pairs, Phys. Rev. D 79 (2009) 115006 [arXiv:0903.1377] [INSPIRE].

[27] U. Ellwanger, J.F. Gunion, C. Hugonie and S. Moretti, Towards a no lose theorem for NMSSM Higgs discovery at the LHC, hep-ph/0305109 [INSPIRE].

[28] U. Ellwanger, Higgs pair production in the NMSSM at the LHC, JHEP 08 (2013) 077 [arXiv: 1306.5541] [INSPIRE].

[29] CMS collaboration, Search for a very light NMSSM Higgs boson produced in decays of the $125 \mathrm{GeV}$ scalar boson and decaying into $\tau$ leptons in pp collisions at $\sqrt{s}=8 \mathrm{TeV}$, JHEP 01 (2016) 079 [arXiv : 1510.06534] [INSPIRE].

[30] ATLAS collaboration, Search for Higgs bosons decaying to aa in the $\mu \mu \tau \tau$ final state in pp collisions at $\sqrt{s}=8 \mathrm{TeV}$ with the ATLAS experiment, Phys. Rev. D 92 (2015) 052002 [arXiv: 1505.01609] [INSPIRE].

[31] CMS collaboration, A search for pair production of new light bosons decaying into muons, Phys. Lett. B 752 (2016) 146 [arXiv:1506.00424] [INSPIRE].

[32] K. Cheung and T.-J. Hou, Light Pseudoscalar Higgs boson in Neutralino Decays in the Next-to-Minimal Supersymmetric Standard Model, Phys. Lett. B 674 (2009) 54 [arXiv: 0809.1122] [INSPIRE].

[33] D.G. Cerdeño, P. Ghosh, C.B. Park and M. Peiró, Collider signatures of a light NMSSM pseudoscalar in neutralino decays in the light of LHC results, JHEP 02 (2014) 048 [arXiv: 1307.7601] [INSPIRE].

[34] C. Han, D. Kim, S. Munir and M. Park, $\mathcal{O}(1)$ GeV dark matter in SUSY and a very light pseudoscalar at the LHC, JHEP 07 (2015) 002 [arXiv: 1504.05085] [INSPIRE].

[35] C. Englert, T.S. Roy and M. Spannowsky, Ditau jets in Higgs searches, Phys. Rev. D 84 (2011) 075026 [arXiv: 1106.4545] [INSPIRE].

[36] A. Papaefstathiou, K. Sakurai and M. Takeuchi, Higgs boson to di-tau channel in Chargino-Neutralino searches at the LHC, JHEP 08 (2014) 176 [arXiv:1404.1077] [INSPIRE].

[37] A. Katz, M. Son and B. Tweedie, Ditau-Jet Tagging and Boosted Higgses from a Multi-TeV Resonance, Phys. Rev. D 83 (2011) 114033 [arXiv:1011.4523] [INSPIRE]. 
[38] Particle Data Group collaboration, K.A. Olive et al., Review of Particle Physics, Chin. Phys. C 38 (2014) 090001 [inSPIRE].

[39] U. Ellwanger and C. Hugonie, NMSPEC: A Fortran code for the sparticle and Higgs masses in the NMSSM with GUT scale boundary conditions, Comput. Phys. Commun. 177 (2007) 399 [hep-ph/0612134] [inSPIRE].

[40] D. Das, U. Ellwanger and A.M. Teixeira, NMSDECAY: A Fortran Code for Supersymmetric Particle Decays in the Next-to-Minimal Supersymmetric Standard Model, Comput. Phys. Commun. 183 (2012) 774 [arXiv:1106.5633] [INSPIRE].

[41] M. Muhlleitner, A. Djouadi and Y. Mambrini, SDECAY: A Fortran code for the decays of the supersymmetric particles in the MSSM, Comput. Phys. Commun. 168 (2005) 46 [hep-ph/0311167] [INSPIRE].

[42] Z. Kang, J. Li and T. Li, On Naturalness of the MSSM and NMSSM, JHEP 11 (2012) 024 [arXiv: 1201.5305] [INSPIRE].

[43] J. Bernon, B. Dumont and S. Kraml, Status of Higgs couplings after run 1 of the LHC, Phys. Rev. D 90 (2014) 071301 [arXiv: 1409.1588] [InSPIRE].

[44] J. Bernon and B. Dumont, Lilith: a tool for constraining new physics from Higgs measurements, Eur. Phys. J. C 75 (2015) 440 [arXiv: 1502.04138] [INSPIRE].

[45] I.F. Ginzburg and M. Krawczyk, Symmetries of two Higgs doublet model and CP-violation, Phys. Rev. D 72 (2005) 115013 [hep-ph/0408011] [INSPIRE].

[46] S. Davidson and H.E. Haber, Basis-independent methods for the two-Higgs-doublet model, Phys. Rev. D 72 (2005) 035004 [Erratum ibid. D 72 (2005) 099902] [hep-ph/0504050] [INSPIRE].

[47] H.E. Haber and D. O'Neil, Basis-independent methods for the two-Higgs-doublet model. II. The significance of $\tan \beta$, Phys. Rev. D 74 (2006) 015018 [hep-ph/0602242] [INSPIRE].

[48] U. Ellwanger and M. Rodriguez-Vazquez, Discovery Prospects of a Light Scalar in the NMSSM, JHEP 02 (2016) 096 [arXiv: 1512.04281] [INSPIRE].

[49] Planck collaboration, P.A.R. Ade et al., Planck 2015 results. XIII. Cosmological parameters, arXiv:1502.01589 [INSPIRE].

[50] G. Bélanger, F. Boudjema, C. Hugonie, A. Pukhov and A. Semenov, Relic density of dark matter in the NMSSM, JCAP 09 (2005) 001 [hep-ph/0505142] [INSPIRE].

[51] G. Bélanger, F. Boudjema, A. Pukhov and A. Semenov, MicrOMEGAs 3 : A program for calculating dark matter observables, Comput. Phys. Commun. 185 (2014) 960 [arXiv: 1305.0237] [INSPIRE].

[52] LUX collaboration, D.S. Akerib et al., Improved Limits on Scattering of Weakly Interacting Massive Particles from Reanalysis of 2013 LUX Data, Phys. Rev. Lett. 116 (2016) 161301 [arXiv: 1512.03506] [INSPIRE].

[53] ATLAS collaboration, Search for direct production of charginos, neutralinos and sleptons in final states with two leptons and missing transverse momentum in pp collisions at $\sqrt{s}=8$ TeV with the ATLAS detector, JHEP 05 (2014) 071 [arXiv: 1403.5294] [INSPIRE].

[54] T. Cheng, J. Li, T. Li and Q.-S. Yan, Natural NMSSM confronting with the LHC7-8, Phys. Rev. D 89 (2014) 015015 [arXiv: 1304.3182] [INSPIRE].

[55] J. Guo, Z. Kang, J. Li, T. Li and Y. Liu, Simplified Supersymmetry with Sneutrino LSP at $8 \mathrm{TeV}$ LHC, JHEP 10 (2014) 164 [arXiv:1312.2821] [INSPIRE]. 
[56] CMS collaboration, Search for new physics in events with same-sign dileptons and jets in pp collisions at $\sqrt{s}=8 \mathrm{TeV}$, JHEP 01 (2014) 163 [Erratum ibid. 1501 (2015) 014] [arXiv: 1311.6736] [INSPIRE].

[57] J. Alwall et al., The automated computation of tree-level and next-to-leading order differential cross sections and their matching to parton shower simulations, JHEP 07 (2014) 079 [arXiv: 1405.0301] [INSPIRE].

[58] A. Alloul, N.D. Christensen, C. Degrande, C. Duhr and B. Fuks, FeynRules 2.0 - A complete toolbox for tree-level phenomenology, Comput. Phys. Commun. 185 (2014) 2250 [arXiv: 1310.1921] [INSPIRE].

[59] C. Duhr and B. Fuks, A superspace module for the FeynRules package, Comput. Phys. Commun. 182 (2011) 2404 [arXiv:1102 .4191] [InSPIRE].

[60] C. Degrande, C. Duhr, B. Fuks, D. Grellscheid, O. Mattelaer and T. Reiter, UFO - The Universal FeynRules Output, Comput. Phys. Commun. 183 (2012) 1201 [arXiv:1108.2040] [INSPIRE].

[61] N.D. Christensen et al., A comprehensive approach to new physics simulations, Eur. Phys. J. C 71 (2011) 1541 [arXiv:0906.2474] [INSPIRE].

[62] P.Z. Skands et al., SUSY Les Houches accord: Interfacing SUSY spectrum calculators, decay packages and event generators, JHEP 07 (2004) 036 [hep-ph/0311123] [INSPIRE].

[63] B.C. Allanach et al., SUSY Les Houches Accord 2, Comput. Phys. Commun. 180 (2009) 8 [arXiv:0801.0045] [INSPIRE].

[64] J.M. Butterworth et al., THE TOOLS AND Monte Carlo WORKING GROUP Summary Report from the Les Houches 2009 Workshop on TeV Colliders, in Physics at TeV colliders, Proceedings, 6th Workshop, dedicated to Thomas Binoth, Les Houches, France, June 8-26 2009, (2010) arXiv: 1003.1643 [INSPIRE].

[65] T. Sjöstrand, S. Mrenna and P.Z. Skands, PYTHIA 6.4 Physics and Manual, JHEP 05 (2006) 026 [hep-ph/0603175] [inSPIRE].

[66] S. Jadach, Z. Was, R. Decker and J.H. Kuhn, The $\tau$ decay library TAUOLA: Version 2.4, Comput. Phys. Commun. 76 (1993) 361 [inSPIRE].

[67] N. Davidson, G. Nanava, T. Przedzinski, E. Richter-Was and Z. Was, Universal Interface of TAUOLA Technical and Physics Documentation, Comput. Phys. Commun. 183 (2012) 821 [arXiv: 1002.0543] [INSPIRE].

[68] DELPHES 3 collaboration, J. de Favereau et al., DELPHES 3, A modular framework for fast simulation of a generic collider experiment, JHEP 02 (2014) 057 [arXiv:1307.6346] [INSPIRE].

[69] M. Cacciari, G.P. Salam and G. Soyez, The anti-k $k_{t}$ jet clustering algorithm, JHEP 04 (2008) 063 [arXiv: 0802.1189] [INSPIRE].

[70] M. Cacciari, G.P. Salam and G. Soyez, FastJet User Manual, Eur. Phys. J. C 72 (2012) 1896 [arXiv: 1111.6097] [INSPIRE].

[71] ATLAS collaboration, Search for the Standard Model Higgs boson in the $H$ to $\tau^{+} \tau^{-}$decay mode in $\sqrt{s}=7 \mathrm{TeV}$ pp collisions with ATLAS, JHEP 09 (2012) 070 [arXiv:1206.5971] [INSPIRE].

[72] J. Li and A.G. Williams, Tau reconstruction methods at an electron-positron collider in the search for new physics, Phys. Rev. D 93 (2016) 075019 [arXiv:1508.05675] [InSPIRE]. 
[73] ATLAS collaboration, Identification and energy calibration of hadronically decaying tau leptons with the ATLAS experiment in pp collisions at $\sqrt{s}=8 \mathrm{TeV}$, Eur. Phys. J. C 75 (2015) 303 [arXiv:1412.7086] [INSPIRE].

[74] J.-H. Kim, Rest Frame Subjet Algorithm With SISCone Jet For Fully Hadronic Decaying Higgs Search, Phys. Rev. D 83 (2011) 011502 [arXiv:1011.1493] [InSPIRE].

[75] J. Thaler and K. Van Tilburg, Identifying Boosted Objects with N-subjettiness, JHEP 03 (2011) 015 [arXiv: 1011.2268] [INSPIRE].

[76] J. Debove, B. Fuks and M. Klasen, Model-independent analysis of gaugino-pair production in polarized and unpolarized hadron collisions, Phys. Rev. D 78 (2008) 074020 [arXiv:0804.0423] [INSPIRE].

[77] J. Debove, B. Fuks and M. Klasen, Transverse-momentum resummation for gaugino-pair production at hadron colliders, Phys. Lett. B 688 (2010) 208 [arXiv:0907.1105] [INSPIRE].

[78] J. Debove, B. Fuks and M. Klasen, Joint Resummation for Gaugino Pair Production at Hadron Colliders, Nucl. Phys. B 849 (2011) 64 [arXiv:1102.4422] [InSPIRE].

[79] J. Debove, B. Fuks and M. Klasen, Threshold resummation for gaugino pair production at hadron colliders, Nucl. Phys. B 842 (2011) 51 [arXiv: 1005.2909] [InSPIRE].

[80] B. Fuks, M. Klasen, D.R. Lamprea and M. Rothering, Gaugino production in proton-proton collisions at a center-of-mass energy of 8 TeV, JHEP 10 (2012) 081 [arXiv:1207.2159] [INSPIRE].

[81] B. Fuks, M. Klasen, D.R. Lamprea and M. Rothering, Precision predictions for electroweak superpartner production at hadron colliders with Resummino, Eur. Phys. J. C 73 (2013) 2480 [arXiv: 1304.0790] [INSPIRE].

[82] W. Beenakker, M. Klasen, M. Krämer, T. Plehn, M. Spira and P.M. Zerwas, The production of charginos/neutralinos and sleptons at hadron colliders, Phys. Rev. Lett. 83 (1999) 3780 [Erratum ibid. 100 (2008) 029901] [hep-ph/9906298] [INSPIRE].

[83] ATLAS collaboration, Measurement of the $t \bar{t}$ production cross-section in pp collisions at $\sqrt{s}=13$ TeV using e $\mu$ events with b-tagged jets, ATLAS-CONF-2015-033 (2015).

[84] J.M. Campbell, R.K. Ellis and C. Williams, Vector boson pair production at the LHC, JHEP 07 (2011) 018 [arXiv: 1105.0020] [InSPIRE].

[85] C.G. Lester and D.J. Summers, Measuring masses of semiinvisibly decaying particles pair produced at hadron colliders, Phys. Lett. B 463 (1999) 99 [hep-ph/9906349] [INSPIRE].

[86] H.-C. Cheng and Z. Han, Minimal Kinematic Constraints and m(T2), JHEP 12 (2008) 063 [arXiv:0810.5178] [INSPIRE]. 